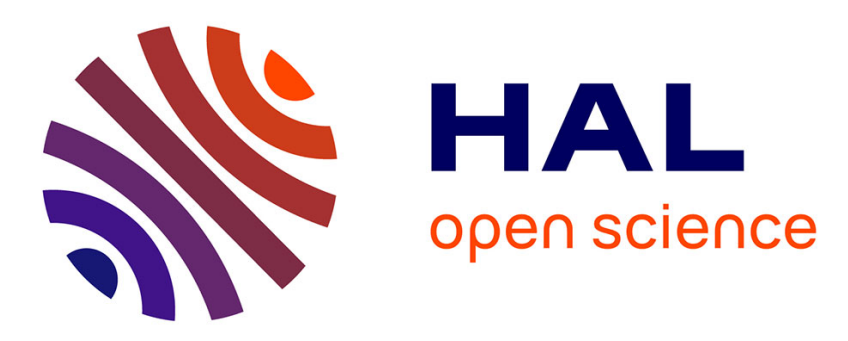

\title{
Anticipation of Brain Shift in Deep Brain Stimulation Automatic Planning
}

Noura Hamzé, Alexandre Bilger, Christian Duriez, Stéphane Cotin, Caroline Essert

\section{- To cite this version:}

Noura Hamzé, Alexandre Bilger, Christian Duriez, Stéphane Cotin, Caroline Essert. Anticipation of Brain Shift in Deep Brain Stimulation Automatic Planning. IEEE Engineering in Medicine and Biology Society (EMBC'15), Aug 2015, Milan, Italy. pp.3635 - 3638 10.1109/EMBC.2015.7319180 . hal-01242851

\section{HAL Id: hal-01242851 \\ https://inria.hal.science/hal-01242851}

Submitted on 14 Dec 2015

HAL is a multi-disciplinary open access archive for the deposit and dissemination of scientific research documents, whether they are published or not. The documents may come from teaching and research institutions in France or abroad, or from public or private research centers.
L'archive ouverte pluridisciplinaire HAL, est destinée au dépôt et à la diffusion de documents scientifiques de niveau recherche, publiés ou non, émanant des établissements d'enseignement et de recherche français ou étrangers, des laboratoires publics ou privés. 


\title{
Anticipation of brain shift in Deep Brain Stimulation automatic planning
}

\author{
Noura Hamzé ${ }^{1,4}$, Alexandre Bilger ${ }^{2}$, Christian Duriez $^{3}$, Stéphane Cotin ${ }^{3,4}$ and Caroline Essert ${ }^{1,4}$
}

\begin{abstract}
Deep Brain Stimulation is a neurosurgery procedure consisting in implanting an electrode in a deep structure of the brain. This intervention requires a preoperative planning phase, with a millimetric accuracy, in which surgeons decide the best placement of the electrode depending on a set of surgical rules. However, brain tissues may deform during the surgery because of the brain shift phenomenon, leading the electrode to mistake the target, or moreover to damage a vital anatomical structure. In this paper, we present a patient-specific automatic planning approach for DBS procedures which accounts for brain deformation. Our approach couples an optimization algorithm with FEM based brain shift simulation. The system was tested successfully on a patient-specific 3D model, and was compared to a planning without considering brain shift. The obtained results point out the importance of performing planning in dynamic conditions.
\end{abstract}

\section{INTRODUCTION}

Deep Brain Stimulation (DBS) is a neurosurgery procedure used to treat a variety of neurological disorders, most commonly Parkinson's disease, dystonia, or essential tremors. It consists in implanting an electrode in a precise brain target, generally the subthalamic nucleus, to stimulate it permanently. The stimulation is done via a neurostimulator, implanted under the patient's chest skin, that delivers electrical impulses through an extension wire to metallic leads located at the tip of the electrode.

The success of the intervention and the reduction of the postoperative symptoms depend on an accurate placement of the stimulating leads at the target location.

Moreover, a misplacement of the electrode may lead to the stimulation of other structures causing side effects, or a massive hemorrhage if the electrode meets a vessel.

Neurosurgeons use MRI and CT images to perform a rigorous preoperative planning: they identify and locate the target within the brain, and trace the trajectory of the electrode. The trajectory planning is subjected to a set of surgical rules to ensure both the safety of the patient and the effectiveness of the procedure. In fact, this preoperative planning step is time-consuming since it is still performed manually in most DBS centers. During the surgery, a burr hole is drilled in the patient's skull, and when the skull and the dura mater

\footnotetext{
*This work was supported by the French National Research Agency (ANR) through the ACouStiC project grant (ANR 2010 BLAN 0209 02)

${ }^{1}$ Noura Hamzé and Caroline Essert are with ICube, University of Strasbourg, France $\{$ hamze, essert $\}$ eunistra.fr

${ }^{2}$ Alexandre Bilger is with the University of Luxembourg, Luxembourg alexandre.bilger@uni.lu

${ }^{3}$ Christian Duriez and Stéphane Cotin are with INRIA, France \{christian.duriez, stephane.cotin\}@inria.fr

${ }^{4}$ Noura Hamzé, Caroline Essert and Stéphane Cotin are affiliated with the Institut Hospitalo-Universitaire de Strasbourg, France
}

are open, cerebrospinal fluid (CSF) may leak through the hole leading to a brain deformation called brain shift. This phenomenon, which is hard to accurately anticipate, can effectively alter the desired results of the intervention. This is due to the difference between the preoperative planning in static condition where no deformation is considered, and the intra-operative dynamic condition where the brain deforms.

In this paper, we address DBS planning in dynamic conditions. We present a patient-specific method which proposes automatically an optimized safe electrode trajectory for DBS procedures and accounts for brain deformation.

\section{RELATED WORKS}

Several techniques can be adpoted for modeling the behaviour of brain tissue. A mass-spring-damper model is used when fast computation is preferred to accuracy, for instance for real-time interaction. For a precise planning, a more accurate model is a better choice. It means a higher computational time which can be acceptable, but needs to remain compatible with clinical use.

Most of the physics-based brain deformation models are based on FEM method, but differ from each other by the choice of the constitutive equation. [10] use a linear constitutive equation, while [12] introduced a non-linear hyperelastic model. The law determines the tissue behaviour, but there is no consensus about a constitutive equation unifying all the applications, such as simulating deformation during a car crash or a neurosurgery. Brain deformation models have been widely used for training systems, registration of medical images [10], or compensating brain shift [4], but not for the preoperative planning of a surgery.

Due to the impossibility to predict CSF loss, [4] precomputed different brain deformations for possible input parameters, but the goal was to update the different structures position during the surgery which is not our case.

In DBS procedures, surgeons usually plan the interventions manually based on MRI and CT images, with the assistance of some interactive medical image visualization workstations such as Medtronics StealthStation or CranialVault [6]. Despite their valuable assistance, the planning process remains mentally difficult and time-consuming. In the last decade, automatic planning solvers were introduced in many works. Some approaches maximize the distance between the trajectory and obstacle structures [3], [14], whereas others take into account a larger variety of placement rules classified into hard or soft constraints to optimize the trajectory [2], [8], [15], and even to perform automatic targeting [6], [11]. 
To the best of our knowledge, no study investigated DBS automatic trajectory planning accounting for brain deformation. We propose a hybrid simulation/planning system to address this problem.

\section{METHODS}

\section{A. Brain model and brain shift simulation}

1) Brain deformation: The brain is considered as a soft body subject to the laws of continuum mechanics. One of the numerical methods to solve the governing equations of motion is the Finite Element Method (FEM). In this work, we use $\mathcal{P}_{1}$ Lagrange tetrahedral elements.

As the brain shift process occurs at a very low velocity, we consider the problem as quasi-static and only look for the configuration of the brain at that equilibrium, disregarding the dynamic transient effects. Finally, the discrete equation to solve is

$$
\mathbf{f}(\mathbf{x})=0
$$

where $\mathrm{x}$ and $\mathbf{f}$ are respectively the position and the force vectors on the nodes of the tetrahedral elements. Here, $\mathrm{f}$ is a nonlinear function of the position of the nodes $\mathbf{x}$, and represents the sum of the internal and external forces. To solve this nonlinear equation, we use a first-order linearization at each time step: $\mathbf{f}(\mathbf{x}+d \mathbf{x})=\mathbf{f}(\mathbf{x})+\mathbf{K}(\mathbf{x}) d \mathbf{x}$, where the Jacobian matrix $\mathbf{K}(\mathbf{x})=\frac{\partial \mathbf{f}}{\partial \mathbf{x}}$ depends on the nodes position. This matrix is called stiffness matrix for the internal forces. The solution of Equation 1 is then approximated with the first iteration of the Newton-Raphson algorithm. The resulting equation is a linear system solved with a Conjugate Gradient algorithm. This process is applied iteratively until reaching equilibrium.

Regarding the application of the simulation, brain deformation can be considered as small. This allows us to use Hooke's law to define the tissue behavior. It defines a linear relationship between strain and stress. From this law, we can write the local (relative to an element $e$ ) stiffness matrix $\mathbf{K}_{\mathbf{e}}=\int_{v_{e}} \mathbf{J}_{\mathbf{e}}{ }^{T} \mathbf{D}_{\mathbf{e}} \mathbf{J}_{\mathbf{e}} d V$ where $v_{e}$ is the volume of element $e$, $\mathbf{J}_{\mathbf{e}}$ denotes a matrix providing strain-displacement relationship and $\mathbf{D}_{\mathbf{e}}$ stands for the strain-stress relationship. In our case, with Hooke's law, $\mathbf{J}_{\mathbf{e}}$ and $\mathbf{D}_{\mathbf{e}}$ are constant. To handle large displacements (while maintaining small deformation), we use a co-rotational formulation [9], where the geometric non-linearities are approximated with the rotation of the element with respect to its initial configuration. With this approach, the stiffness matrix $\mathbf{K}_{\mathbf{e}}^{\mathbf{r}}$ of the element $e$ is defined as $\mathbf{K}_{\mathbf{e}}^{\mathbf{r}}=\mathbf{R}_{\mathbf{e}}{ }^{T} \mathbf{K}_{\mathbf{e}} \mathbf{R}_{\mathbf{e}}$ where $\mathbf{R}_{\mathbf{e}}$ is the rotation matrix of element $e$. Finally, the global matrix $\mathbf{K}$ is assembled from the local element stiffness matrices $\mathbf{K}_{\mathbf{e}}$.

\section{2) CSF model and boundary conditions:}

a) Interaction with bony structures: When the brain deforms and moves, it may collide the endocranium. Once they have been detected, contacts are solved using Signorini's law $0 \leq \delta \perp \lambda \geq 0$. It establishes an orthogonal relationship between the contact response force $\lambda$ and the interpenetration distance $\delta$. We ensure the Signorini's condition is fulfilled at the end of each time step by adding a term of constraints in Equation 1: $\mathbf{f}(\mathbf{x})=\mathbf{H}^{T} \lambda$ where $\mathbf{H}$ is a matrix containing

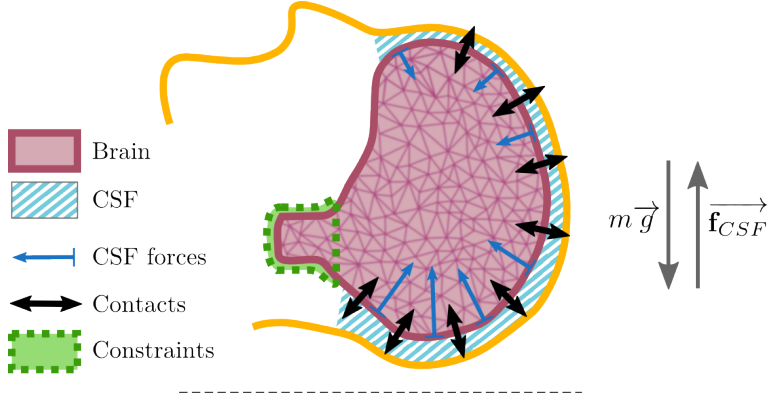

Fig. 1: Illustration showing the components of the simulation

the constraints directions and $\lambda$ is Lagrange multipliers containing the constraint force intensities. $\lambda$ is unknown and has to be computed. A linear complementary system is obtained, and is solved using a Gauss-Seidel algorithm. More details on the overall solving process are given in [7].

b) Cerebro-spinal fluid: The main cause of brain shift is a loss of Cerebro-Spinal Fluid (CSF) surrounding the brain. The density of CSF is similar to water $(\rho=1007$ $\mathrm{kg} / \mathrm{m}^{3}$ ). A loss of CSF leads to a change of pressure inside the skull and causes a deformation of the brain. The action of CSF on the brain is modeled with a hydrostatic pressure:

$$
\mathbf{f}_{C S F}=\int_{S_{e}}\left(\rho g h+p\left(z_{0}\right)\right) \mathrm{d} \mathbf{S}
$$

where $S_{e}$ is the surface of a submerged element belonging to the surface of the brain, $g$ denotes the gravitational acceleration, $h$ stands for the height from a point to the fluid surface, and $p\left(z_{0}\right)$ is the pressure of the point $z_{0}$ located on the fluid surface. Fig.1 illustrates the different components into play in the simulation. The amount of brain shift is controlled by the fluid level. With a loss of CSF the fluid level, $h$, and the fluid forces decrease.

\section{B. Trajectory planning}

Our preoperative planning approach is based on a geometric constraint solving method in static conditions we previously published in [8].

1) static environment: To implement a planning in static conditions $\mathbf{E}_{\mathbf{s}}$, we formalize the surgical rules into two categories of geometric constraints: Hard constraints HC, and soft constraints SC. While $\mathbf{H C}$ express strict rules which must not be violated such as crossing a vessel, SC express preferences such as keeping the trajectory as far as possible from the ventricles. To solve $\mathbf{H C}$, we extract from the initial skin surface $\boldsymbol{\Omega}_{\mathbf{0}}$ the feasible insertion zone $\boldsymbol{\Omega}_{\mathrm{s}}$ which satisfies HC. This is done by eliminating from the skin mesh the triangles that are invisible from the target. Afterwards, we compute an optimal trajectory based on an estimation of its quality in respect to SC. To this end, we assign a cost function $f_{i}$ to each $\mathbf{S C}$, and create a main aggregative objective function $f$ composed from all SC. By minimizing $f$ using a derivative-free optimization technique we obtain an optimal trajectory (insertion point and direction). 


$$
f=\frac{\sum_{i=1}^{n} w_{i} \cdot f_{i}}{\sum_{i=1}^{n} w_{i}}
$$

In Eq. 3, weights $w_{i}$ reflect the importance of each surgical rule relatively to the others and are determined by surgeons.

2) dynamic environment: In order to implement a planning in dynamic conditions $\mathbf{E}_{\mathbf{d}}$, we need to anticipate the potential deformations caused by the brain shift phenomenon $\varphi$. For now, it is not possible to determine preoperatively the exact magnitude of a brain shift. It may vary from a minimum level $\varphi_{0}$ (corresponding to no brain shift) to a maximum $\varphi_{\max }$ (corresponding to full CFS loss through the entry point). However, we can estimate $\varphi_{\max }$ for a given entry point. For this reason, our objective is to find a safe trajectory whatever the brain shift intensity from $\varphi_{0}$ to $\varphi_{\max }$ and including all possible intermediate levels $\varphi_{i}$.

While in $\mathbf{E}_{\mathbf{s}}$ the planning is performed on the initial static model $\mathbf{M}_{\mathbf{s}}$, in $\mathbf{E}_{\mathbf{d}}$ it is computed on several deformed models $\mathbf{M}_{\mathbf{d} \varphi}$ computed as described in Section III-A using SOFA biomechanical simulation framework [1]. We precompute a range of simulations corresponding to levels $\varphi_{0}$ to $\varphi_{\max }$ and store them as a patient-specific deformations database. We access the simulations database during the planning process using our planning plugin implemented in MITK [16].

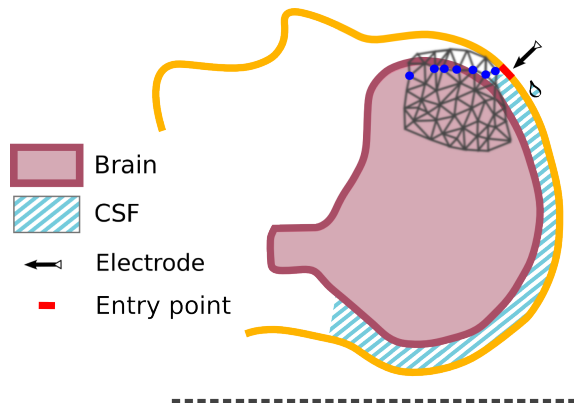

Fig. 2: Schematic representation of the entry points (blue) of the initial skin mesh $\Omega_{0}$ lying at the same height $h$ and likely to lead to the same possible maximal brain shift $\varphi_{\max }$.

Our method consists in two phases:

- Compute the feasible insertion zone in dynamic conditions $\Omega_{\mathrm{d}}$.

- Compute the optimized trajectory in $\Omega_{\mathrm{d}}$.

To obtain $\Omega_{\mathrm{d}}$ we assign a height level $h$ to each entry point $p_{i} \in \boldsymbol{\Omega}_{\mathbf{0}}$ (as illustrated in Fig.2). For each height $h$, we build its corresponding deformed model $\mathbf{M}_{\mathbf{d} h}$ by summing up all the deformable models $\mathbf{M}_{\mathbf{d} \varphi_{0}}$ to $\mathbf{M}_{\mathbf{d} \varphi_{\max }}$ resulting from $\varphi_{0}$ to $\varphi_{\max }$. After that, we check whether $p_{i}$ satisfies $\mathbf{H C}$ on $\mathbf{M}_{\mathbf{d} h}$ to add it to $\boldsymbol{\Omega}_{\mathbf{d}}$, otherwise we exclude it.

In a second step, we apply the Nelder-Mead optimization method [13] over $\Omega_{\mathrm{d}}$ to find the best trajectory. At each iteration, we propose a candidate trajectory $\tau$, get the model $\mathbf{M}_{\mathbf{d} h}$ depending on the candidate entry point's height $h$, and evaluate $f(\tau)$ on $\mathbf{M}_{\mathbf{d} h}$. The iterations are stopped when the improvement of $f$ falls under a threshold $\varepsilon$, then $\tau$ is stored as the optimal trajectory.

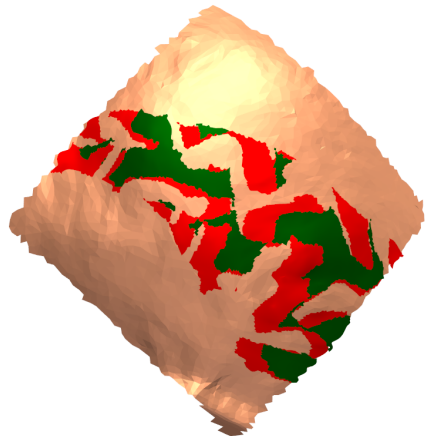

Fig. 3: $\boldsymbol{\Omega}_{\mathbf{0}}$ is the large rectangular patch, $\boldsymbol{\Omega}_{\mathrm{s}}$ is a subset of $\boldsymbol{\Omega}_{0}$ and is the union of red and green shapes, and $\boldsymbol{\Omega}_{\mathrm{d}}$ is a subset of $\boldsymbol{\Omega}_{\mathrm{s}}$ and is the green mesh.

\section{EXPERIMENTAL RESULTS}

We implemented our method on a patient-specific 3D brain model obtained thanks to pyDBS [5] pipeline. The model consists of surface meshes of the sulci, the ventricles, the subthalamic nucleus, and a skin patch. A volumetric model is built for the simulation part. Blood vessels being difficult to segment from MR images and knowing that they are embedded in the sulci that are more visible, we considered that the sulci form with the ventricles the anatomical structures to be avoided as obstacles. The target of the stimulation is the center of the subthalamic nucleus, and the initial solution space is the skin patch. The number of elements in the model is $83 \mathrm{k}$, and the number of simulations in the patient-specific deformations database file is 20 . The Lamé's parameters were set to $\lambda=1291 \mathrm{~Pa}$ and $\mu=1034 \mathrm{~Pa}$ according to [10]. Experiments were performed on an Intel Core i7 running at $2.67 \mathrm{GHz}$ with 8GB RAM workstation.

Firstly, we compared both feasible insertion zones $\boldsymbol{\Omega}_{\mathrm{s}}$ and $\boldsymbol{\Omega}_{\mathrm{d}}$ computed in $\mathbf{E}_{\mathrm{s}}$ and $\mathbf{E}_{\mathrm{d}}$ respectively. The obtained results are shown on Table I.

TABLE I: Insertion zones: sizes, computation times, and coverage ratio.

\begin{tabular}{lccc}
\hline \hline & $\boldsymbol{\Omega}_{\mathbf{0}}$ & $\boldsymbol{\Omega}_{\mathbf{s}}$ & $\boldsymbol{\Omega}_{\mathbf{d}}$ \\
\hline \# Triangles & 67920 & 17408 & 7868 \\
Comp. time (s) & - & 12 & 36 \\
$\boldsymbol{\Omega}_{\mathbf{0}}$ coverage (\%) & 100 & 25.6 & 11.6 \\
\hline \hline
\end{tabular}

The values in Table I show that $\boldsymbol{\Omega}_{\mathrm{d}}$ is smaller than $\Omega_{\mathrm{s}}$ which was quite expected due to the larger number of obstacles in $\mathbf{E}_{\mathrm{d}}$ than in $\mathbf{E}_{\mathrm{s}}$. The percentage of $\boldsymbol{\Omega}_{\mathrm{d}}$ to $\boldsymbol{\Omega}_{\mathrm{s}}$ is equal to $45.2 \%$ which means that the feasible insertion zone is reduced by $54.8 \%$. A visual illustration of the coverage ratio is shown on Fig.3. It can be also noticed that the required time to build $\boldsymbol{\Omega}_{\mathrm{d}}$ is around $36 \mathrm{~s}$ which is three times as much as the time required to build $\boldsymbol{\Omega}_{\mathrm{s}}$, but still keeps the approach compatible with clinical use.

Secondly, we compared the optimization results in both $\mathbf{E}_{\mathbf{s}}$ and $\mathbf{E}_{\mathbf{d}}$. Table II shows the performance of Nelder-Mead 


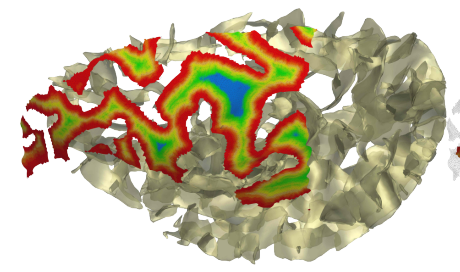

(a) $\mathbf{E}_{\mathbf{s}}, \mathbf{M}_{\mathbf{s}}: \omega_{\mathbf{s}}$ on $\boldsymbol{\Omega}_{\mathbf{s}}$

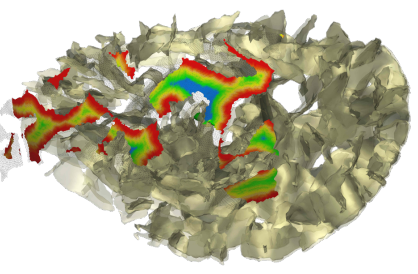

(b) $\mathbf{E}_{\mathbf{d}}, \mathbf{M}_{\mathbf{d}}: \omega_{\mathrm{s}}$ on $\boldsymbol{\Omega}_{\mathbf{d}}$
Fig. 4: (a) distance map to the borders of $\Omega_{\mathrm{s}}$, and (b) the same distance map projected onto $\Omega_{\mathrm{d}}$. Parts are cut even in the initially safe (blue) zone.

optimization algorithm in both $\mathbf{E}_{\mathbf{s}}$ and $\mathbf{E}_{\mathbf{d}}$. We report the value of the evaluation of the objective cost function $f$ which we are minimizing, the distance between the optimized computed trajectory and the obstacles (ventricles and sulci), the number of iterations, and at last the convergence time.

TABLE II: Nelder-Mead performance in $\mathbf{E}_{\mathbf{s}}$ and $\mathbf{E}_{\mathbf{d}}$

\begin{tabular}{lcc}
\hline \hline Nelder-Mead & $\mathbf{E}_{\mathbf{s}}$ & $\mathbf{E}_{\mathbf{d}}$ \\
\hline eval $(f)[0,1]$ & 0.28 & 0.38 \\
dist. from ventricles (mm) & 11.87 & 7.39 \\
dist. from sulci (mm) & 5.13 & 3.12 \\
\# of iterations & 31 & 21 \\
time (s) & 0.034 & 0.258 \\
\hline \hline
\end{tabular}

The values in Table II show that the optimization algorithm used in $\mathbf{E}_{\mathbf{s}}$ could also converge in $\mathbf{E}_{\mathbf{d}}$, and find a trajectory sufficiently safe for DBS interventions even in case of a brain shift. The optimized trajectory in $\mathbf{E}_{\mathbf{d}}$ was closer to the obstacles than the one in $\mathbf{E}_{\mathbf{s}}$ but remains far enough from them. Consequently, the best evaluation value in $\mathbf{E}_{\mathbf{d}}$ (0.38) was not as good as the best one in $\mathbf{E}_{\mathbf{s}}(0.28)$ but can be considered as acceptable for clinical practice. The optimization time is negligible in both cases and compatible with a use in clinical routine.

An interesting observation we obtain is that the safest insertion regions in static conditions shown as blue zones on Fig.4a can be withdrawn from the set of safe trajectories in case of brain shift, as shown on Fig.4b where some formerly blue parts are cropped.

\section{CONCLusion}

We presented a novel approach for DBS automatic preoperative planning coupling physical simulations with geometric optimization to help the surgeon to anticipate a possible deformation during the planning. We tested our system on a patient-specific 3D model, with very promising results.

The obtained results illustrate the large variation of size and shape of the safe insertion zones for DBS interventions between the static and the dynamic conditions, as it was shrunk of more than $50 \%$ when considering a possible brain shift. This variation shows the interest of including the brain shift prediction during the planning phase to remove dangerous entry points that would not be detected otherwise. Although avoiding a sum of deformations from no brain shift to a maximum possible brain shift to anticipate a wide range of possible levels of deformations causes a high restriction of the safe insertion zone, we could still find in the remaining insertion zone an optimized entry point which is safe and efficient in a reasonable computation time.

Further works could be done to improve the system: on the first hand, improving the accuracy of the brain shift model by using more complex deformation and fluid models, and on the other hand different optimization techniques could be investigated and compared. Finally, further clinical validation is required to assess the quality of the overall system.

\section{REFERENCES}

[1] SOFA, Simulation Open Framework Architecture. Http://www.sofaframework.org/

[2] Bériault, S., Subaie, F., Mok, K., Sadikot, A., Pike, G.: Automatic trajectory planning of DBS neurosurgery from multi-modal MRI datasets. In: proceedings of MICCAI'11, Springer LNCS, vol. 6891, pp. 259-266 (2011)

[3] Brunenberg, E., Vilanova, A., Visser-Vandewalle, V., Temel, Y., Ackermans, L., Platel, B., ter Haar Romeny, B.: Automatic trajectory planning for deep brain stimulation: A feasibility study. In: proceedings of MICCAI'07, Springer LNCS, vol. 4791, pp. 584-592 (2007)

[4] Chen, I., Coffey, A.M., Ding, S., Dumpuri, P., Dawant, B.M., Thompson, R.C., Miga, M.I.: Intraoperative brain shift compensation: accounting for dural septa. IEEE transactions on bio-medical engineering 58(3), 499-508 (2011)

[5] D'Albis, T., Haegelen, C., Essert, C., Fernandez-Vidal, S., Lalys, F., Jannin, P.: PyDBS: an automated image processing workflow for deep brain stimulation surgery. International Journal of Computer Assisted Radiology and Surgery pp. 1-12 (2014)

[6] D'Haese, P.F., Pallavaram, S., Li, R., Remple, M.S., Kao, C., Neimat, J.S., Konrad, P.E., Dawant, B.M.: Cranialvault and its crave tools: A clinical computer assistance system for deep brain stimulation (DBS) therapy. Medical Image Analysis 16(3), 744-753 (2012)

[7] Duriez, C., Cotin, S., Lenoir, J., Neumann, P.: New approaches to catheter navigation for interventional radiology simulation. Computer Aided Surgery 11(6), 300-308 (2006)

[8] Essert, C., Haegelen, C., Lalys, F., Abadie, A., Jannin, P.: Automatic computation of electrode trajectories for deep brain stimulation: a hybrid symbolic and numerical approach. International journal of computer assisted radiology and surgery 7(4), 517-532 (2012)

[9] Felippa, C., Haugen, B.: A unified formulation of small-strain corotational finite elements: I. theory. Computer Methods in Applied Mechanics and Engineering 194(21), 2285-2335 (2005)

[10] Ferrant, M., Nabavi, A., Macq, B., Black, P.M., Jolesz, F.a., Kikinis, R., Warfield, S.K.: Serial registration of intraoperative MR images of the brain. Medical image analysis 6(4), 337-59 (2002)

[11] Guo, T., Parrent, A., Peters, T.: Automatic target and trajectory identification for deep brain stimulation (DBS) procedures. In: proceedings of MICCAI'07, Springer LNCS, vol. 4791, pp. 483-490 (2007)

[12] Miller, K., Chinzei, K.: Mechanical properties of brain tissue in tension. Journal of biomechanics 35, 483-490 (2002)

[13] Nelder, J., Mead, R.: A simplex method for function minimization. Computer Journal 7(4), 308-313 (1965)

[14] Shamir, R., Tamir, I., Dabool, E., Joskowicz, L., Shoshan, Y.: A method for planning safe trajectories in image-guided keyhole neurosurgery. In: proceedings of MICCAI'10, vol. 6363, pp. 457-464. Springer LNCS (2010)

[15] Tirelli, P., de Momi, E., Borghese, N., Ferrigno, G.: An intelligent atlas-based planning system for keyhole neurosurgery. In: Computer Assisted Radiology and Surgery supplemental, pp. S85-S91 (2009)

[16] Wolf, I., Vetter, M., Wegner, I., Bttger, T., Nolden, M., Schbinger, M., Hastenteufel, M., Kunert, T., Meinzer, H.P.: The medical imaging interaction toolkit. Medical Image Analysis 9(6), 594-604 (2005) 